\title{
Automatic Order Management System for Restaurants
}

\author{
Rohith $^{1}$, Swathi N Rao ${ }^{2}$, Sweeda Noronha ${ }^{3}$, Ujwala J Shetty ${ }^{4}$, Wilson Samuel Mathias ${ }^{5}$, \\ Dr. Balachandra Achar $\mathbf{H ~ V}^{6}$ \\ Student, ECE, SMVITM, Udupi, India ${ }^{1,2,3,4,5}$ \\ Head of the department, ECE, SMVITM, Udupi, India ${ }^{6}$
}

\begin{abstract}
This paper is about a technology that provides quick ordering system inside the restaurant using restaurant's $\mathrm{Wi}-\mathrm{Fi}$ and about providing internet access to the authenticated customers. As customer gets connected to the hotel Wi$\mathrm{Fi}$, a page will be displayed to enter table number for authentication purpose. During this time, he/she cannot access any sites other than hotel's menu page. A unique password will be assigned and displayed on the LCD display mounted on respective table. As he/she completes the authentication, the menu page will be displayed on his/her phone, through which the customer can place the order. As the customer selects the dishes, it will be stored in the cart, from which he/she can verify again and modify the quantity details and then place the order. After placing the first order the network will allow the customer to access other sites. It also provides paperless billing system to the customer and also keeps update of the current status of billing for the customer's benefit. The ordered information is displayed on the display in the kitchen through Wi-Fi for faster servicing without any delay. The servicing is done by the restaurant personnel. Once the payment is done by the customer, the ordered data will be stored in separate database and the customer's internet access privilege will be cancelled.
\end{abstract}

Keywords: Wi-Fi, Web page, Controller, Authenticate, Database, Networking.

\section{INTRODUCTION}

Nowadays importance is given to the wireless technology and automation system. To match the world's fast-growing scenario of emerging technologies there is a need for the innovative methods in every aspect of the life creating wide opportunities. Digital India campaign has been launched by the Government of India which aims at smart cities with the intension of binding every field with the smarter technologies. Earlier, costlier interface devices like touchpad's and PDAs (Personal Digital Assistants) were used, which makes installation costlier. Application based services needs to be pre-installed; this also annoys the customers with advertisements. Online ordering systems through web pages which require internet service in customer's device also proved inefficient in maximizing the ordering experience.

In this paper, we have eliminated all these disadvantages and made it more efficient. The system we are presenting is not an app and thus no need to install or update anything on phone hence reduces memory utilization. PDAs consume lot much space on the table and also updating and maintaining it is also time consuming and expensive, this can be overcome in this paper. This system also reduces the usage of Wi-Fi as authorized users can only access it, thus building a good business strategy. It also satisfies the customers, as customer gets the knowledge about the exact price and the exact picture of the dish he has selected, as the page designed is attractive and easy to use.

\section{LITERATURE SURVEY}

Initially the restaurants were atomised with the limited range RF transmitter for food ordering system, which has the transmission range of only 4-5 meters, this was overcome by the technology proposed in [1]. According to this concept of an automatic self-ordering system, order is directly given to the chefs by the customer. The real time ordered data is sent wirelessly using Zigbee technology. Chef can get the information about the order on the display screen along with the table number.

In previous technology, use of microcontroller unit on table increases the complexity and PDA system to the waiter increases the Cost of installation and maintenance. LAN system from server to chef increases the networking complexity. Hence this was improved in [2]. This paper aims to implement a restaurant ordering system which enables each customer to wirelessly order his own choice of food straight from the e-menu shown on an embedded touch screen on each customer table without bothering any staff and send the order straight to the kitchen. The whole food process can also be monitored via this touch screen. 


\section{ISO 3297:2007 Certified}

Vol. 5, Issue 6, June 2017

Touch screens on the table occupy large space and need more Maintenance. It is also costlier for installation. Due to the emerging trends of android devices the developers concentrated more on android applications. Therefore, the menu systems came in android apps in [3], Each table in the restaurant will be accompanied with an android tablet or a Smartphone. The device will be loaded with an android supporting application containing food menu. The wireless connection is created using Wi-Fi network.

In [4], they proposed a system based on both java and android, customer order the food by using android based their own touchpad or touchscreen device. The interface is designed using the java. The kitchen is also equipped with the screens to inform the order placed. The network covers the servicing area, kitchen and cashier desk. Automatic billing generated is displayed on the respected device on the table itself. This paper gave the java as solution for networking and introduced Automatic bill generation.

According to the previous paper we have to download restaurant app and install it using internet. Hence in [5], gave a solution. According to them system was implemented using android application for Tablet PC's on the table provided by the restaurant. The front end will be developed using JAVA Android and the backend will work on MySQL database. The ordering system is done through the tablet placed on the table by the restaurant. This paper gave a good data management operation using MySQL Database which is open source.

There is also a development in an interest based application in [6], suggested that the employers/students can place the order by selecting the nearby canteen/cafeteria through the android application pre-installed in their smart phones. The food is served to the designated place by the canteen personals. This will help in saving the time of the employers/students and they can get fresh and healthy food in their place only. As android apps consume the space in mobile memory and such third-party apps will irritate with advertisements. The service gets increased.

In paper [7] gave a stable technology to implement the networking concept by the authorization mechanism. Wireless networks are to have a captive portal protecting the network from unauthorized users and for auditing the authorized users. In this paper, we will describe how captive portals help authorize the internet access by recognizing MAC address of the users. Public wireless networks are common and you can often find them in coffee shops, in schools, on trains or in other public environments. However, in many cases the owners of these networks want to control the access to the networks. A captive portal is basically a system that holds an unauthenticated user captive until some sort of web based authentication mechanism authenticates the user.

In paper [8] using firewall HTTP and HTTPS request will be intercepted and redirected to the authenticator. He proposes a dual-stack Lightweight Stateless HTTP Redirector (LSHR) as a dedicated web server to perform redirection service inside Firewall and The User Session Manager (USM) keeps track of all active users' sessions. IPv4 and IPv6 addresses from the same login session are stored as a single entry in the database along with common hash code. The same authentication page authenticates for both IP4 and IP6 addresses bind with common hash code.

\section{METHODOLOGY}

When a client enters the hotel he/she will get Wi-Fi option of the hotel on his/her phone. By connecting to the hotel Wi-Fi his/her phone gets connected to a wireless network which is controlled by the server of the hotel. Authentication page is displayed, where customer has to enter the table number. As soon as table number is entered a particular pass key is generated and stored in database, the same will be displayed on the respective LCD display mounted on that table which has to be entered in the phone to recognize the fake ordering. Controller which continuously monitors the database configures the displays with respect to the table.

Once the verification is done, the web page (menu page) is displayed. By using categorized dish item in database, he/she can add any number of item to his/her order list by adding it to his/her cart which displays the number of item added, which is stored in the database of the server. when clicked on an item name another page containing the image of the dish with price and editable quantity field will be displayed. Customer can view histher cart by clicking on the cart option and modify the quantity and delete the dish before ordering.

As he/she orders, amount gets updated and total amount is displayed in ordered list. Once he/she places the order he/she is free to access the internet of the restaurant which will be authenticated by the firewall till his dining. At the same time the cook inside the kitchen comes to know about the ordered dish via interface display placed in the kitchen, which continuously monitors the database. After each payment, the ordered data like dish name and quantity are stored 


\section{International Journal of Innovative Research in} Electrical, Electronics, Instrumentation and Control Engineering

\section{ISO 3297:2007 Certified}

Vol. 5, Issue 6, June 2017

in the database. This is further used to keep track of how many dishes are served at the end of the day. The ordered information is displayed on the display in the kitchen through wireless $\mathrm{Wi}-\mathrm{Fi}$, to fasten the servicing. The servicing is done by the restaurant personnel's in order to increase the dining experience of the customers.

\section{BLOCK DIAGRAM}

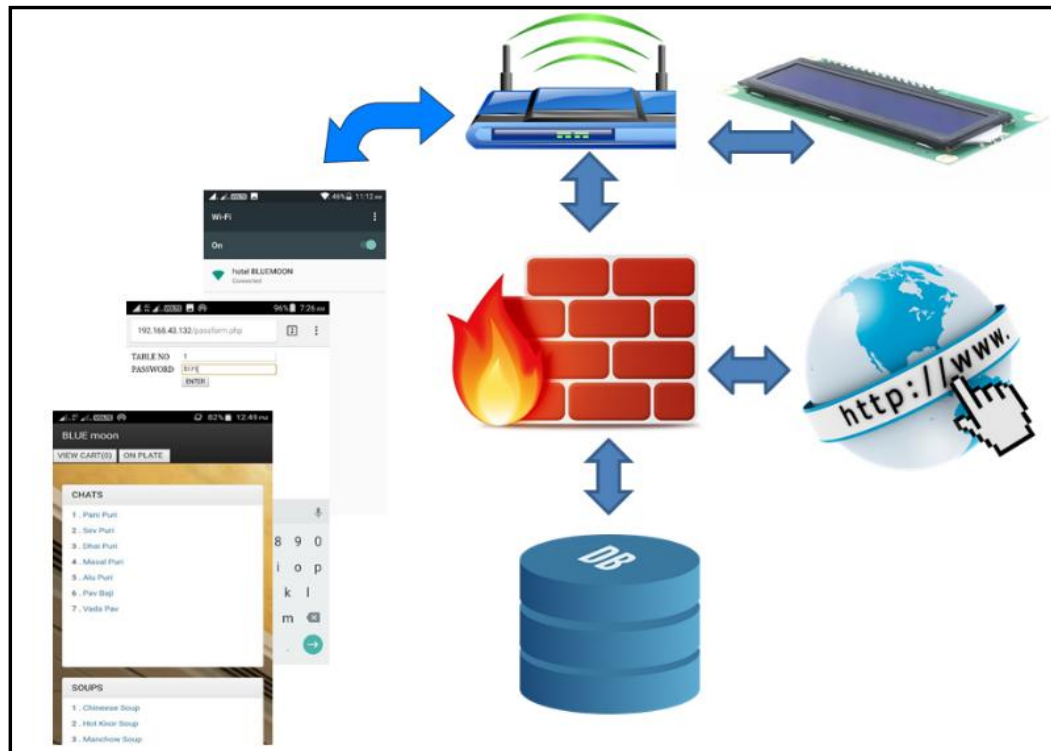

Fig.1.Block diagram of the system

\section{FLOW CHART}

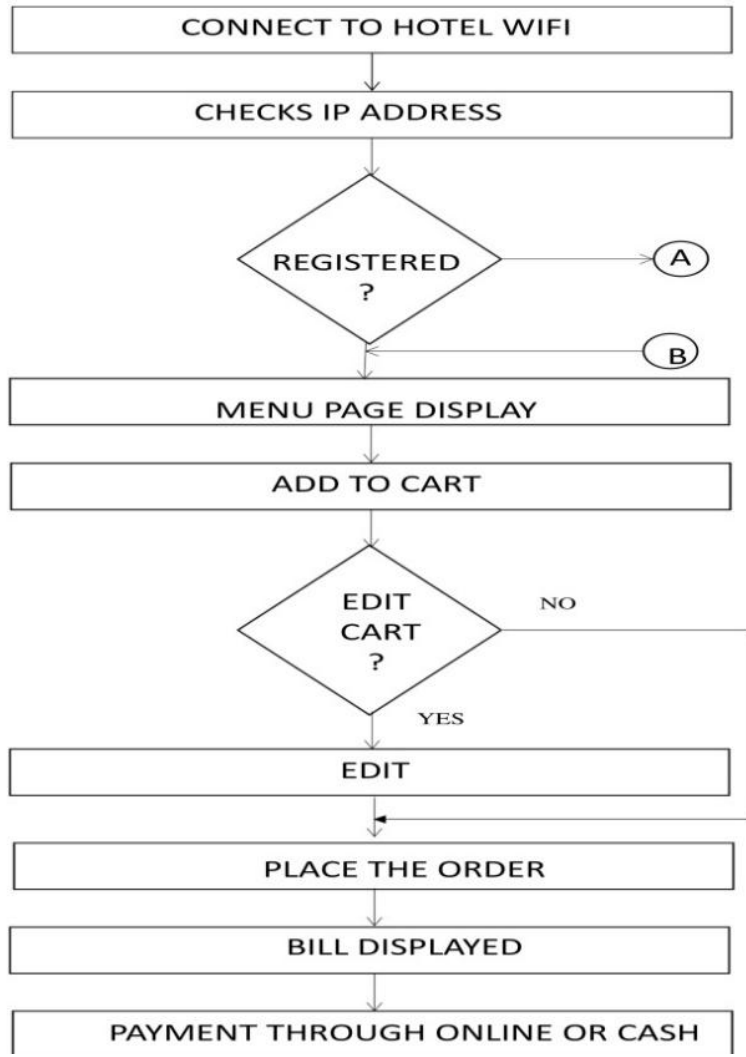



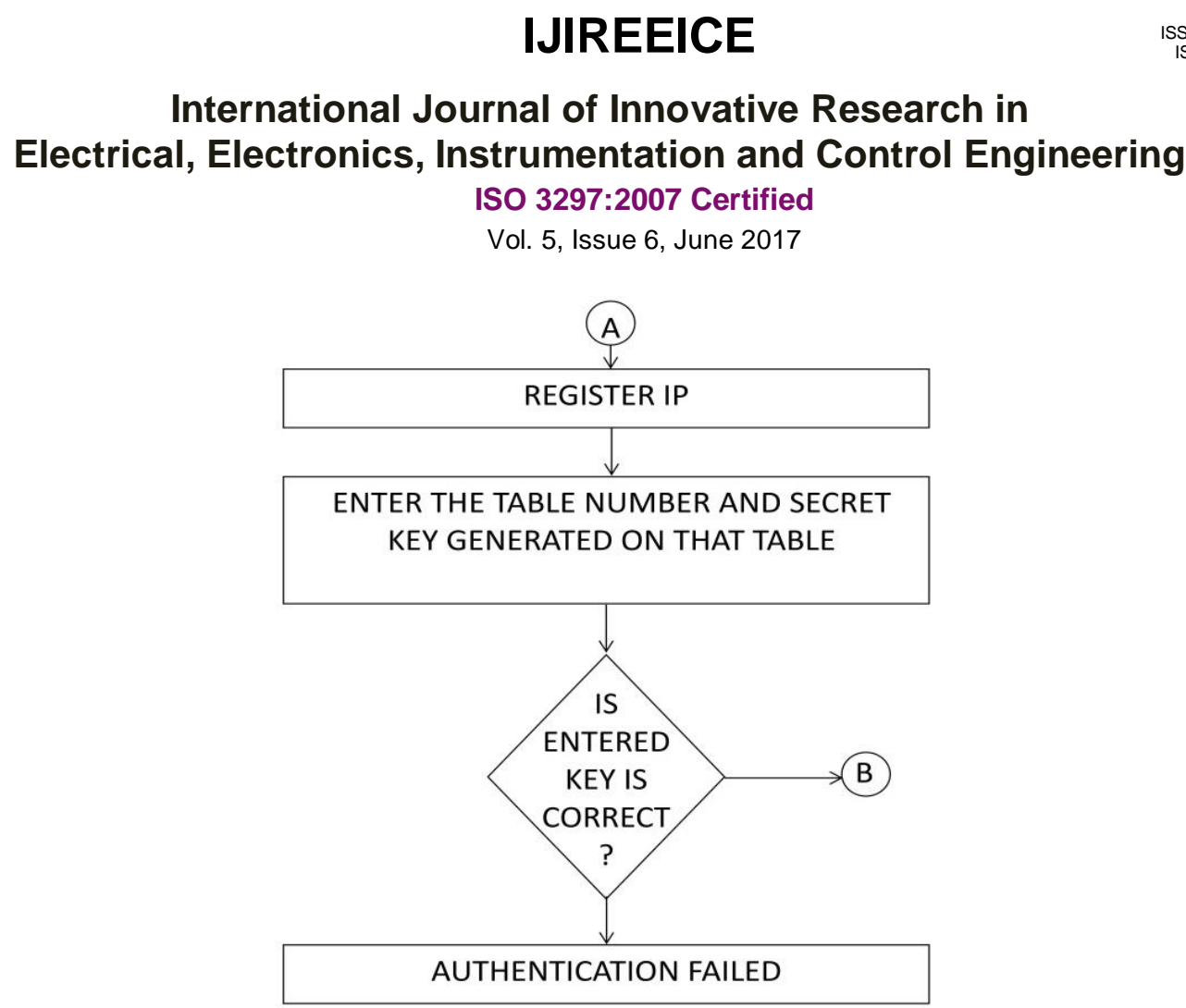

Fig 2. Flowchart of proposed system

\section{RESULT}

Hotel menu is hosted from the server personal computer through hotel router. Each table is equipped with the LCD interfaced with Raspberry Pi to display verification code as shown in fig 3.

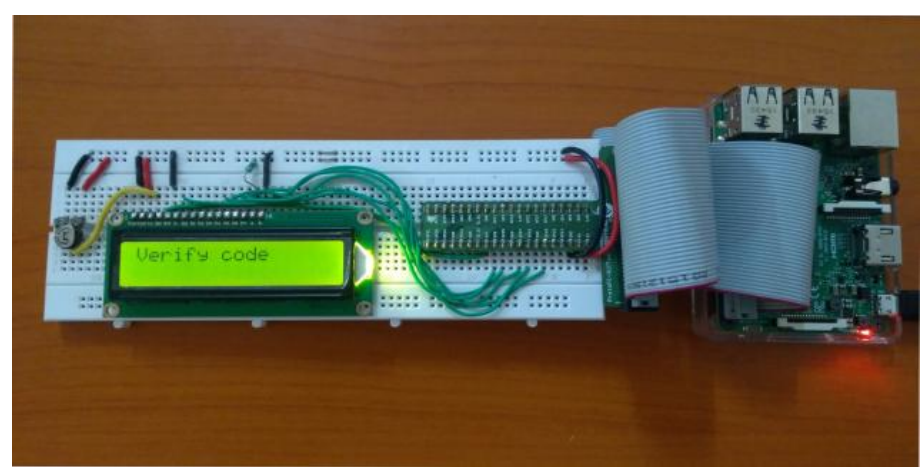

Fig.3 Interfaced LCD display with Raspberry Pi

As soon as customer enters the table number in his device a unique 4-digit code will be generated for customers IP address and displayed as shown in Fig 4 on respective table.

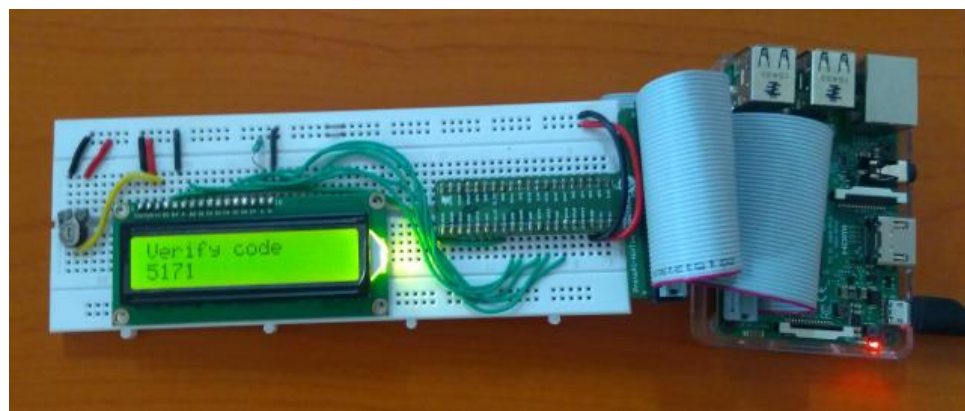

Fig 4 Display showing verification code

Once customer is authenticated by entering code as shown in fig 5 , then he/she can use the menu page for ordering as in fig 6 . 


\section{IJIREEICE}

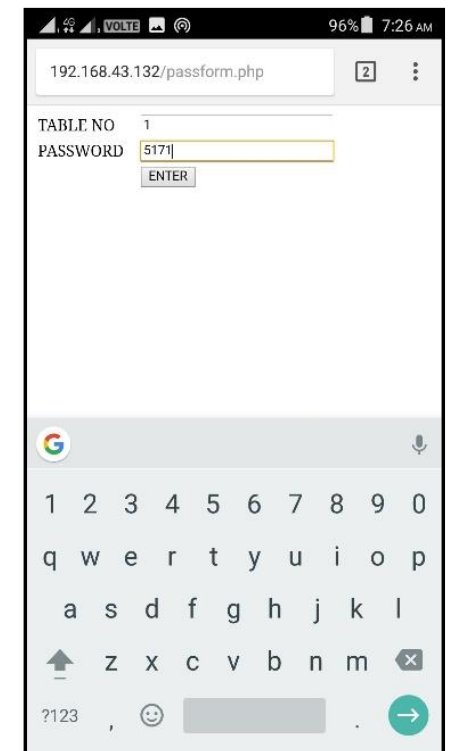

Fig 5. Entering authentication code

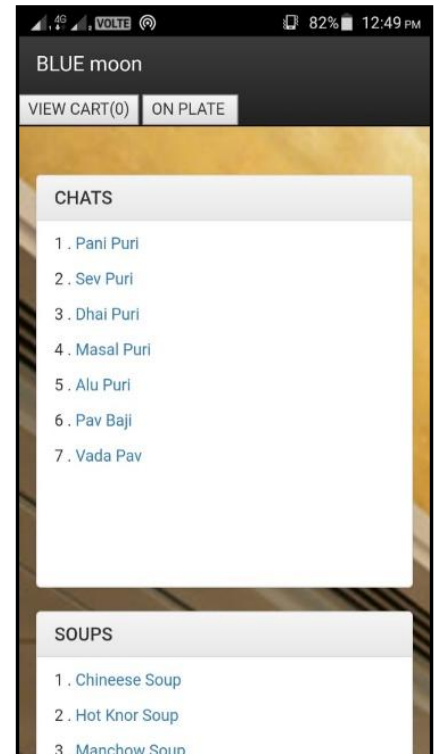

Fig 6. Menu page displayed on mobile

By using configured dish item in database, he/she can add any number of item to his/her order list by adding it to his/her cart which displays the number of item added with editable quantity field as in fig 7. He/She can also view his/her ordered list with price (fig 8). In kitchen ordered dishes are displayed as in fig 9.

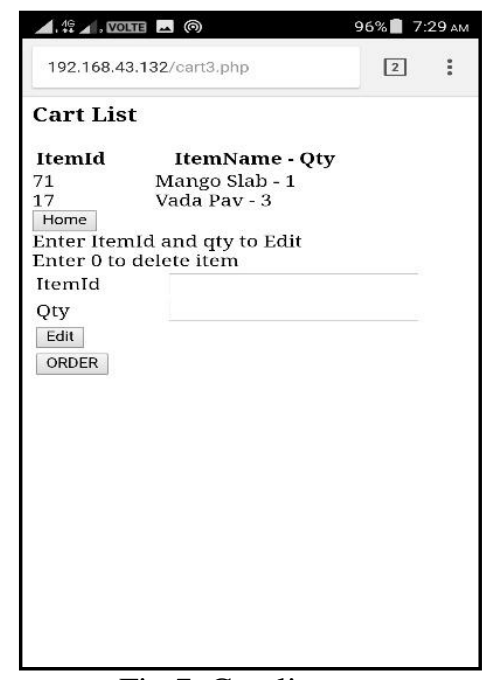

Fig 7. Cart list

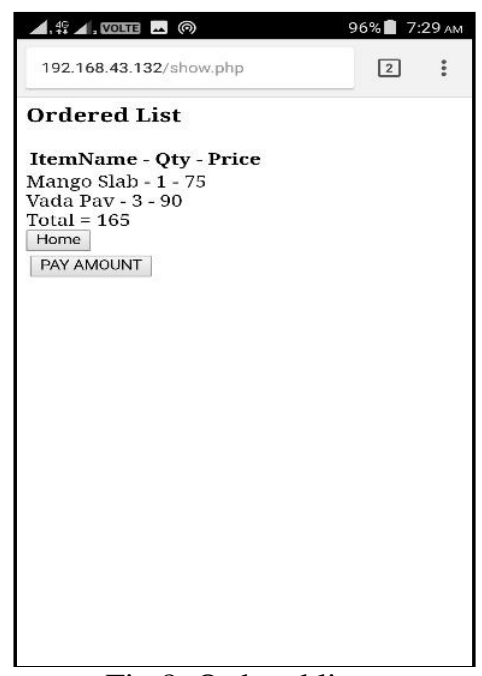

Fig 8. Ordered list

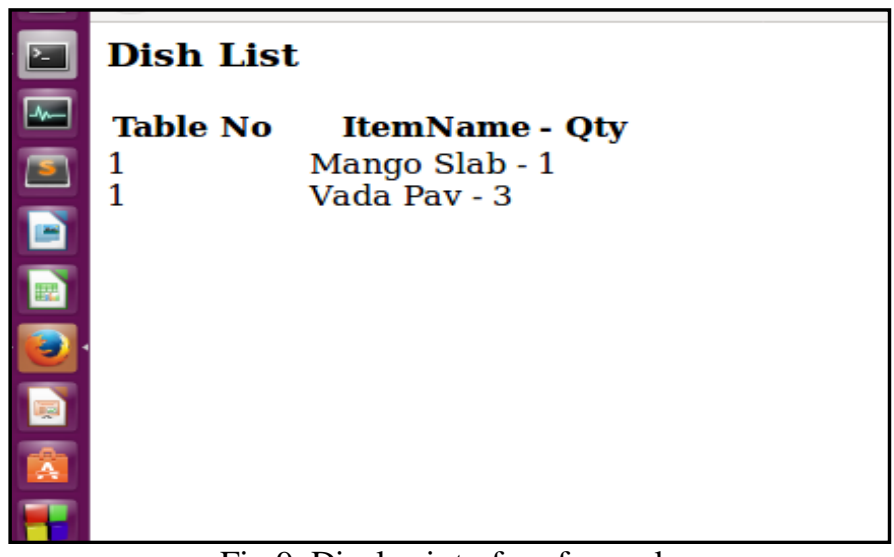

Fig 9. Display interface for cook 


\section{IJIREEICE

\section{CONCLUSION}

Technology is such a term which can change the complete operation of a particular system. In today's world, we find that each and every field is based on the use of some kind of technology. Customer requirements are very necessary while considering the hotel business. If we analyze the different types of customer requirements, we will find that they are almost the same. The customer needs a good service, good quality food, less time consumption, no confusion in placing and receiving an order, quick billing with no errors. All this can be made possible in our system. This system provides a better solution to overcome the disadvantages in the previous technologies. It is a zero-delay system. And the customer need not install any application on his phone, hence eliminates advertisements which interrupts the mobile operations. Customer need not have mobile data on his phone. The operation is completely using hotel Wi-Fi. And also, he/she need not give any personal information since the system takes the IP address of the phone for the process. Once the operation is completed that is once the customer pays his bill the IP address stored is deleted from the database. In the existing technologies installation is costlier since they have PDA's on every table. But for our system investment is less since every customer will be using his personal phones. And here we have an authentication process to avoid false ordering. During authentication, a password is generated which will be displayed on the table. This password will be unique for each table number and it is generated randomly. In this system, customer can use free internet only after the order is placed.

\section{REFERENCES}

[1] Sheifali Gupta,Ph.D.Shivam Gupta, Sourav Garg, Nitin Goyal,Sukhbeer Singh , "Chef Alerting System using Wireless Zigbee Technology" , International Journal of Applied Information Systems (IJAIS), Volume 6- No. 7, January 2014.

[2] B.Vinodhini, K.Abinaya, R.Roja, M.Rajeshwari, "Wireless Two-way Restaurant Ordering System via Touch Screen", The International Journal Of Engineering And Science (IJES), Volume 3, Issue 7, March 2014.

[3] Mayur D. Jakhete, Piyush C. Mankar, "Implementation of Smart Restaurant with e-menu Card", International Journal of Computer Applications (0975 - 8887) Volume 119 - No.21, June 2015.

[4] Kirti Bhandge, Tejas Shinde, Dheeraj Ingale, Neeraj Solanki, Reshma Totare, "A Proposed System for Touchpad Based Food Ordering SystemUsing Android Application”, International Journal of Advanced Research in Computer Science \& Technology (IJARC ST 2015), Vol. 3, Issue 1, (Jan. - Mar. 2015).

[5] Ashutosh Bhargave, Niranjan Jadhav, Apurva Joshi, Prachi Oke, Prof. Mr. S. R Lahane, “ DigitalOrdering System for Restaurant UsingAndroid", International Journal of Scientific and Research Publications, Volume 3, Issue 4, April 2013.

[6] Abhishek Singh, Amit Tanwar, Aditya Sawant, Chaitanya Parulekar, Kunal Yadav, "Canteen Food Ordering Android System", International Journal on Recent and Innovation Trends in Computing and Communication , Volume: 4 Issue: 4, April 2016.

[7] Jihyuk Choi, Sang-Yoon Chang, Diko Ko, Yih-Chun Hu, "Secure MAC-Layer Protocol for Captive Portals in Wireless Hotspots", A reviewed text paper at the direction of IEEE Communications Society subject matter experts for publication in the IEEE ICC 2011 proceedings2011.

[8] Surasak Sanguanpong, Kasom Koht-Arsa, “A Design and Implementation of Dual-Stack Aware Authentication System for Enterprise Captive Portal", Network and service management (CNSM), 2013, 9th international conference. 